\title{
Annual congress of the EANM 2007, Copenhagen, Denmark Abstracts Tuesday
}

\author{
H. Demir • Y. Z. Tan • S. Isgoren • G. Gorur Daglioz • \\ F. Berk
}

Published online: 20 June 2008

(C) Springer-Verlag 2008

\section{Erratum to: Eur J Nucl Med Mol Imaging (2007) \\ 34 (Suppl 2)224 \\ DOI 10.1007/s00259-007-0545-8}

On page S224 the wrong abstract was published as number 476. The correct abstract is given below:

\section{6}

Comparison of exercise and pharmacological stress gated SPECT in detecting transient left ventricular dysfunction

H. Demir, Y. Z. Tan, S. Isgoren, G. Gorur Daglioz, F. Berk; Kocaeli University School of Medicine, Kocaeli, TURKEY. Aim: Transient left ventricular dysfunction (TLVD) is observed due to post-exercise stunning in patients with coronary artery disease (CAD). Pharmacological stimulation differs from exercise stress since it does not cause demand ischaemia. The aim of this study was to determine whether TLVD could also be seen after pharmacological stress (dipyridamole).

Methods: Four hundred thirty-nine subjects with known or suspected CAD were included to the study. Gated single photon emission computed tomography (GSPECT) was performed for all patients after exercise $(n=220$, group I) or pharmacological stress $(n=219$, group II) according to two days (stress-rest) protocol. Stress, rest and difference (stress-rest value) left ventricular ejection fractions (LVEF), were derived automatically. Summed stress score (SSS), summed rest score (SRS) and summed difference score (SDS) for myocardial perfusion was calculated using a 20segment model and a 5-point scoring system. An SDS $>3$ was considered ischemia. Based on perfusion findings,

The online version of the original can be found at http://dx.doi.org/ 10.1007/s00259-007-0545-8.

H. Demir $(\bowtie) \cdot$ Y. Z. Tan · S. Isgoren · G. Gorur Daglioz • F. Berk Kocaeli University School of Medicine,

Kocaeli, Turkey

e-mail: hakandemir99@yahoo.com patients were subdivided into normal (group A) and ischaemia group (group B).

Results: Stress induced ischaemia was observed in 223/439 patients $(50.8 \%)$. Mean stress, rest and difference LVEF values and perfusion scores were summarized in Table I. For group B, LVEF was significantly decreased after stress compared with rest values $(\mathrm{p}=0.000$ for group $\mathrm{BI}$ and $\mathrm{BII})$. But, in group $\mathrm{A}$, the difference between stress and rest LVEF values was not significant ( $p=0.670$ and $p=0.200$ for group AI and AII, respectively). TLVD ( $\geq 5 \%$ for difference LVEF) was observed in $81 / 223$ patients (36\%) and in 20/ $216(9 \%)$ patients in group B and A, respectively $(\mathrm{p}=$ 0.000). In group II, TLVD was detected in 35/104 (34\%) and $8 / 115(7 \%)$ patients with and without ishaemia, respectively $(p=0.000)$. On the other hand, in group I, we found TLVD in 46/119 (39\%) and 12/101 (12\%) patients in patients with and without ishaemia, respectively $(p=0.000)$.

Table I. Mean LVEF values and perfusion scores

\begin{tabular}{lllllll}
\hline \multicolumn{5}{c}{ All } & \multicolumn{5}{c}{ Group I } & \multicolumn{3}{c}{ Group II } \\
& Patients & & & & & \\
& A & B & A & B & A & B \\
Stress LVEF & $64.15 \pm$ & $53.24 \pm$ & $64.52 \pm$ & $53.98 \pm$ & $63.82 \pm$ & $52.38 \pm$ \\
$(\%)$ & 11.78 & 14.80 & 9.36 & 13.88 & 13.59 & 15.80 \\
Rest LVEF & $63.98 \pm$ & $56.59 \pm$ & $64.69 \pm$ & $57.60 \pm$ & $63.35 \pm$ & $55.43 \pm$ \\
$(\%)$ & 11.79 & 15.39 & 9.85 & 13.28 & 13.27 & 16.56 \\
D. LVEF & $0.17 \pm$ & $-3.35 \pm$ & $-0.17 \pm$ & $-3.61 \pm$ & $0.47 \pm$ & $-3.05 \pm$ \\
$(\%)$ & 3.93 & 4.39 & 3.96 & 4.54 & 3.90 & 4.20 \\
SSS & 0.0 & 7.0 & 0.0 & 7.0 & 0.0 & 7.0 \\
SRS & 0.0 & 1.0 & 0.0 & 1.0 & 0.0 & 2.0 \\
SDS & 0.0 & 5.0 & 0.0 & 5.0 & 0.0 & 5.0 \\
\hline
\end{tabular}

D: difference

Conclusion: In conclusion dipyridamole is believed to be less likely than exercise to induce ischemia. However, in this study, TLVD after stress was observed following not only post-exercise but also pharmacological stress consistent with ischemia. 